Research Article

\title{
Traffic Information Collection Using Wireless Sensor Network Positioning Technology
}

\author{
Jinxi Zhang $\mathbb{D},{ }^{1}$ Wenying Zhu, ${ }^{1}$ Xueying $\mathrm{Wu}^{2}$ and Tianshan $\mathrm{Ma}^{2}$ \\ ${ }^{1}$ College of Transportation Engineering, Chang'an University, Xi'an 710064, China \\ ${ }^{2}$ School of Economics and Management, Chang'an University, Xi'an 710064, China \\ Correspondence should be addressed to Jinxi Zhang; 2018023008@chd.edu.cn
}

Received 5 June 2021; Revised 12 August 2021; Accepted 19 August 2021; Published 6 September 2021

Academic Editor: Haibin Lv

Copyright (c) 2021 Jinxi Zhang et al. This is an open access article distributed under the Creative Commons Attribution License, which permits unrestricted use, distribution, and reproduction in any medium, provided the original work is properly cited.

\begin{abstract}
The wireless sensor network integrates sensor technology, microelectromechanical technology, distributed information processing technology, and wireless communication technology. In order to solve this problem, this paper designs and proposes an anchor node self-location algorithm. Aiming at the positioning accuracy of wireless sensor network nodes, this paper proposes an improved algorithm for sensor network node positioning that uses error correction methods to reduce accumulated distance errors and positioning errors. In this paper, the designed routing algorithm is simulated and implemented, and the performance of the routing algorithm is evaluated based on different network topologies. From the analysis results, compared with the existing typical routing algorithms, the routing algorithms designed in this paper can effectively reduce the energy consumption of the network and prolong the lifetime of the network. The core of the algorithm is to integrate the known and available information of the system to locate unknown anchor nodes. This greatly reduces the number of anchor nodes whose initial position information is required by the system, and under the condition of less impact on the positioning accuracy of the system, the cost of the system is reduced and the scope of application of the system is improved. This paper has deeply studied the positioning and tracking problems in wireless sensor networks, including node positioning, biochemical gas source positioning, and target tracking, and designed and developed a platform for positioning and tracking application research to lay the foundation for further application research. In the study of the above problems, new methods of positioning and tracking with theoretical and practical value are proposed for different practical application scenarios, and the performance is verified and evaluated through computer simulation.
\end{abstract}

\section{Introduction}

In recent years, wireless technology and sensor technology have achieved unprecedented development, people's demand for related technologies is increasing day by day, and a new type of wireless sensor network technology turned out [1]. This technology is widely used in military, industry, agriculture, animal husbandry, smart home, smart city, and many other fields by virtue of the low cost, small size, and easy deployment of sensor nodes [2]. Although these applications cover all walks of life and have their own characteristics, they have one thing in common, that is, they need the location information of the sensor nodes [3]. If the location information of the sensor node is missing, then all the information obtained by the system loses its meaning. Therefore, the way to obtain node location information is particularly important. For a long time, the acquisition of sensor location information has been done through GPS positioning devices [4]. We know that the application of GPS devices requires a lot of money. This does not meet our low-cost requirements for sensor nodes. Therefore, how to control the cost of locating sensor nodes is a hot topic at present [5].

When wireless sensor networks are used in indoor environments or cities, a type of problem often occurs, that is, the transmission of information between sensor nodes will be blocked by obstacles, making the system unable to communicate in line-of-sight. The direct consequence of this kind of problem is to make the information we get through the positioning node have a large deviation and reduce the system positioning performance [6]. Aiming at such 
problems, this paper proposes a nonline-of-sight identification algorithm. The algorithm uses the feature of nonlineof-sight to make part of the information change significantly, identifies the node that cannot communicate with line-of-sight from the system, and removes the node during positioning, thereby eliminating the impact of nonline-ofsight problem on system positioning performance.

In this paper, wireless sensor networks are applied to the location of biochemical gas sources to improve people's ability to respond quickly to emergencies. (1) A robust maximum likelihood location algorithm is proposed, and the direct trilateral method and the nonlinear least square method are compared. (2) In order to eliminate the inherent shortcomings of the centralized positioning algorithm and save network energy, the hierarchical sensor network is applied to biochemical source positioning, and a distributed positioning algorithm based on improved particle filtering under the hierarchical sensor network is proposed, which manages nodes through subregions. The state transfers, and the loop iterative estimation obtains the convergent biochemical gas source position. Many R\&D institutions are committed to developing a new type of sensor node location information acquisition technology. At present, the method of relying on the wireless sensor network itself for positioning is quite popular. (3) The special feature of this technology is that it only needs to use the communication information between nodes and adopt a special algorithm to determine the position of the node to complete the positioning. This article, respectively, introduces the related concepts of sensor network positioning technology, several positioning algorithms, and the development status, so as to analyze the current problems of the technology and propose improvements.

\section{Related Work}

The above positioning technology mainly focuses on the study of the situation where all the nodes in the sensor network are static nodes. Part of the literature began to study the location method of mobile nodes in the sensor network. Zhou et al. [7] were the first to use a single mobile beacon point combined with RSSI measurement technology to estimate the node location. Sugano et al. [8] introduced the denoising method in the image domain to the network location and proposed a node location method based on distributed nonparametric Kernel estimation, combined with TOA ranging technology to get the node location. Liu et al. [9] proposed a coarse-grained distance-independent positioning algorithm that uses radio-frequency connectivity constraints to reduce the unknown uncertainty of unknown nodes. Vançin and Erdem [10] applied rigidity theory to estimate the location of unknown nodes by mobile nodes, solved the unknown nodes by a geometric method, and selected four received beacon points distributed on concentric circles, and the vertical bisector is between the two beacon points. The intersection is the unknown node position. Jo et al. [11] used the sequential Monte Carlo (SMC) method to locate each movement node of the mobile sensor network in the literature. The author proposes a positioning scheme called LandScape for outdoor WSN, which uses Unscented Kalman Filter (UKF) to estimate the position of the node.

Target tracking in WSN adopts collaborative processing between nodes with known locations in the network to locate the position of the moving target at each time and obtain the moving trajectory of the moving target. Compared with traditional tracking methods, although WSN is used for target positioning and tracking, it faces many challenges, its advantages are also obvious. In the article, Nabeel et al. [12] analyzed in detail the three main phases of the target tracking process based on sensor networks, namely, detection, location, and notification phases. The sensor nodes detect and track the target in a cooperative manner, notify the nodes located near the predicted trajectory of the target to join the tracking process, and, at the same time, restrict communication to the nodes near the target and its future movement route. Oppermann et al. [13] proposed a target tracking scheme based on binary sensor nodes. Each node cannot detect the distance to the target, but can determine the circular area containing the target and the duration of the target in the area. Through the cooperation of multiple nodes, an overlapping coverage area is the target location. This scheme obtains the trajectory of the target by deploying a large number of simple and cheap sensor nodes, but the position of each node needs to be known, and the time between nodes needs to be synchronized. Some scholars have proposed a message-driven sensor query mechanism IDSQ (information-driven sensor querying). The core idea is based on the Bayesian algorithm, which uses the information measured by the sensor nodes to iteratively estimate the target position. Bayesian classification is a very simple classification algorithm, and its basic idea is simple: in terms of text classification, it believes that the relationship between two words in the bag of words is independent of each other, that is, the feature vector of an object Each dimension is independent of each other. The selection of a single tracking head node for information fusion at the next moment in the algorithm is based on information gain indicators, such as Mahalanobis distance, interactive information, and information entrapment. This algorithm only activates one head node at each positioning moment, thereby greatly reducing the number of nodes participating in tracking and positioning, reducing the energy consumption of the entire network, but it also reduces the fault tolerance and robustness of the network [14-16]. Some researchers have also proposed a dual space method to track a continuous moving surface target, such as the spread tracking of wildfires, the tracking of typhoon paths, and the tracking of the spread of biochemical substances. They proposed a "location center" method for collaborative sensing and target positioning. The current node estimates the direction of the target and warns of the fixedly divided cells that the target will enter. The nodes in the early warning unit cooperate to detect the appearance of the target, and when the classifier judges that it is the expected target type, the tracking program is started [17].

In addition, some scholars have also proposed some selforganizing distributed sensor network target tracking prediction algorithms, in which the prediction technology uses 
pheromone, Bayesian, and extended Kalman filter technology, related mathematic models as shown in references $[18,19]$. The proposed DCTC (dynamic convoy tree-based collaboration) transmission tree tracking algorithm is based on the research of target tracking from the perspective of network communication. The transmission tree is a dynamic tree structure composed of nodes near the moving target. And some nodes will be dynamically added or deleted as the target moves [20]. The nodes near the moving target carry out cooperative tracking through the transmission tree structure, which reduces the communication overhead between nodes while ensuring efficient tracking of the target. The algorithm relies on the tree structure of the delivery tree itself, emphasizing the network layer domain. Some scholars use the auxiliary particle filter method to solve the fusion problem in target tracking based on the SMC (Sequential Monte Carlo) framework and select the next head node for fusion based on the information utility function of the optimal moisture content. The calculation of the utility function based on the head node selection is extremely complicated, similar to IDSQ. The distributed fusion center is based on a single activation node and uses a distributed particle filter algorithm to predict and track the target. The algorithm uses the EM algorithm to train the Gaussian mixture parameters to make it closer to the expression particle itself. The parameter transfer of the Gaussian mixture model (GMM) replaces the transfer of the particles themselves, which greatly reduces the data communication between the head nodes and reduces energy consumption. In particular, the positioning accuracy needs to be improved, and a large number of particles need to be delivered [21-23].

\section{Construction of Traffic Information Collection Model Using Wireless Sensor Network Positioning Technology}

3.1. Hierarchical Structure of WSN. Wireless sensor networks (WSN) are a new type of network technology that integrates sensor technology, MEMS technology, wireless communication technology, and distributed information processing technology [24-27]. Figure 1 shows the hierarchical distribution of wireless sensor networks.

Through the cooperation between nodes, it can perceive, collect, and process information about the environment or monitored objects in the monitored area in real-time and transmit the processed information to interested network end users.

$$
\begin{aligned}
& (x, y)=\left\{\frac{1}{n} * \sum x(i), \frac{1}{n} * \sum y(i)\right\}, \\
& \left\{\begin{array}{l}
d 1=\sqrt{(x-x 1)^{2}+(y-y 1)^{2}} \\
d 2=\sqrt{(x-x 2)^{2}+(y-y 2)^{2}}
\end{array}\right.
\end{aligned}
$$

WSN is a distributed self-organizing network system composed of a large number of ubiquitous tiny sensor nodes with wireless communication and computing capabilities.
According to the needs of the environment, the designated tasks can be completed through the cooperative perception, collection, processing, and distribution of perception information between these tiny sensor nodes with limited functions.

$$
\left[\begin{array}{l}
x \\
y
\end{array}\right]=[x-x 1 y-y 1]^{-1} *\left[\begin{array}{c}
x^{2}-x 1^{2} \\
y^{2}-y 1^{2}
\end{array}\right]
$$

The system includes three basic elements: sensing objects, sensor nodes, and observers. Wireless network is a communication method between sensors and between sensors and observers and is responsible for establishing communication paths between nodes and observers.

$$
G(i, j)=\left[\begin{array}{cc}
(x-x 1) * \sec ^{2}(i) & 0 \\
0 & (x-x 2) * \sec ^{2}(j)
\end{array}\right] .
$$

Different types of nodes or nodes in different geographical locations in the network form different subnets, and the subnets are connected through gateways (or base stations). The gateway transmits the relevant signals in the WSN to the control center server via satellite, wireless network, or Internet.

$$
u(x, y)=\tan (\theta)=\frac{y(i)-y 0}{x(i)-x 0}, i=1,2, \cdots, n .
$$

Terminal users query and search for relevant data through the server and send corresponding downlink commands to the gateway through the server, and then, the gateway sends to each node in the WSN, which is executed by the nodes to realize remote monitoring and operation of the monitored area.

$p(x(k) \mid k-1)=\int p(x(k) \mid k-1, y) * p(x(k-1) \mid y) * d(x(k-1))$.

Compared with the gateway, the processing capacity, storage capacity, and communication capacity of the node are usually weak, and the energy is limited (usually powered by batteries). Each node can act as a data collector, router, or a combination of several roles, complete the collection and processing of local data, transfer of intermediate data, and cooperate with other nodes to complete specific tasks.

$$
\left\{\begin{array}{l}
S(k)=\left.\frac{d f(x)}{d x}\right|_{x=m(k-1), k-1}, \\
T(k)=\left.\frac{d h(x)}{d x}\right|_{x=m(k-1), k-1} .
\end{array}\right.
$$

The processor is the center of the entire node, responsible for controlling the operation of the entire node, storing and processing the information collected by the node, and other nodes collecting and forwarding information. The 


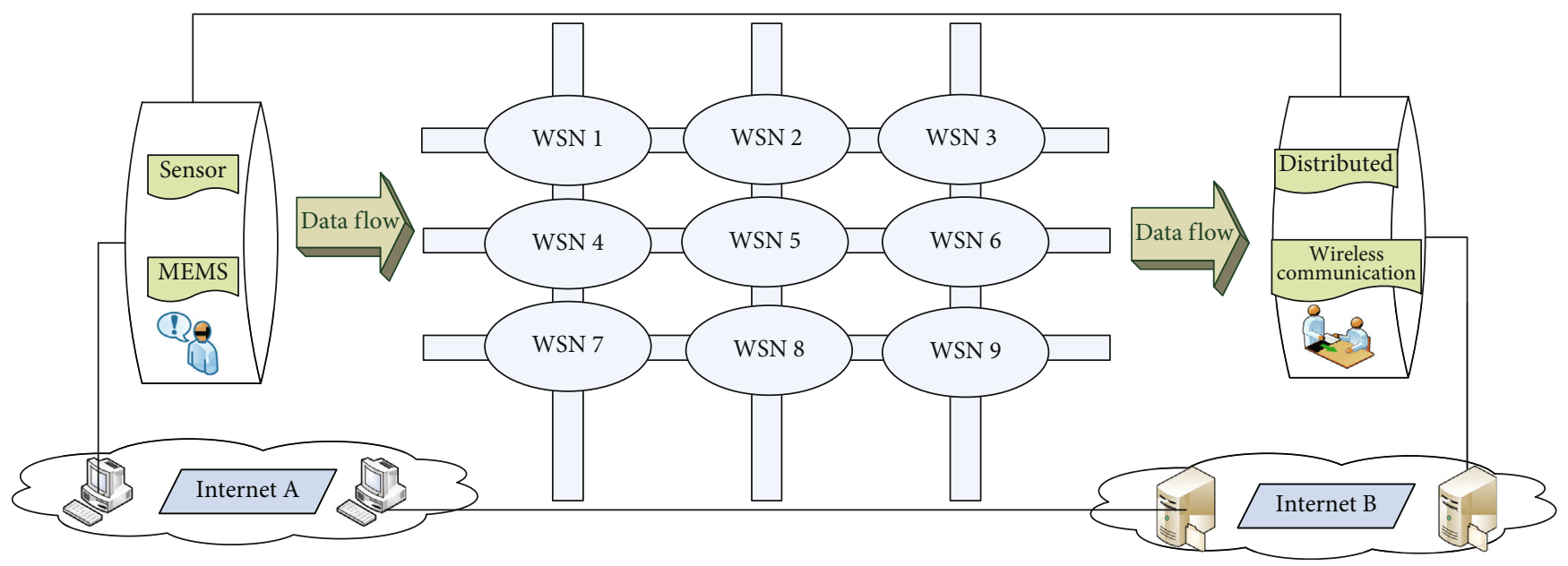

FIGURE 1: Hierarchical distribution of wireless sensor networks.

communication module is responsible for data, control information, and wireless communication between nodes.

$$
\begin{gathered}
\sum_{i=0}^{2 n} w(i) *(x(i)-z)^{T} *(x(i)-z)=0, \\
z(k) \sim q(x(k) \mid x(k-1)) \approx N(\overline{m(k)}, \overline{p(k)}) .
\end{gathered}
$$

The energy supply module is responsible for the energy supply required for the operation of the entire node (the gateway may use continuous power supply, ordinary nodes often use battery power, and for some important nodes, energy regeneration equipment such as photoelectric conversion units may be used). Some nodes contain auxiliary modules.

\subsection{Traffic Information Collection Algorithm. Positioning} and tracking technology in WSN includes two meanings: node self-positioning and moving target positioning and tracking [28-30]. Node self-positioning belongs to the research scope of network middleware technology and belongs to the research part of network system [31, 32]. The purpose is to provide information including node location for network routing layer and higher application layer services. Moving target positioning and tracking belong to the application layer research of WSN, which uses the collaborative signal processing between sensor nodes in the network to complete the positioning and tracking of moving targets [33-35]. According to whether the distance information is measured during the positioning process, the existing positioning algorithms are divided into a range-based algorithm and a range-free algorithm. The data of moving target tracking can be defined as the trajectory of the moving target and the data collected when tracking the target. For example, according to the data of the location and tracking of the sound emitted by the moving target, the position coordinates of the target at each moment can be calculated according to the trajectory of the target, and then the sound emitted from the target after reaching the node is calculated according to the position of the node. Finally, some ran- domly distributed small variables are added to these data to make it more in line with the actual situation. Part of the research involves mobile node-assisted positioning. The following will summarize related types of typical positioning algorithms.

The distance-dependent positioning algorithm measures the distance or angle information between adjacent nodes and uses the measured information to calculate the location of unknown nodes. Figure 2 shows the flow of the traffic information collection algorithm. The measurement technology in the sensor network can be broadly divided into three categories: angle of arrival (AOA), signal strength (Received Signal Strength Indicator, RSSI), and distance measurement correlation. RSSI ranging mechanism based on the received signal strength: it uses the known signal strength of the transmitting point and the signal strength received by the receiving node to calculate the propagation loss of the signal and uses the theoretical or empirical model to convert the propagation loss into a distance meter to measure the distance. However, ranging from RSSI is usually not accurate and unreliable. This is because RF signals are more severely affected by complex environments such as obstructions and shields. Usually, we use sound or radio frequency signal, and its accuracy is higher. AOA ranging mechanism based on signal arrival angle: the receiving node senses the direction of arrival of the transmitting node signal through an antenna array or multiple ultrasonic receivers, calculates the relative position or angle between the receiving node and the transmitting node, and then calculates it by triangulation location of the node. TDO ranging mechanism based on signal arrival time difference: the transmitting node transmits two wireless signals with different propagation speeds at the same time, and the receiving node calculates the distance between the two nodes based on the time difference between the arrival of the two different signals and the known rate of the two signals. Sound signals and radiofrequency signals are often used. Because sound transmission is simple and predictable in open areas, this technology can achieve high accuracy and low cost. For example, only MIC and speakers are required. 


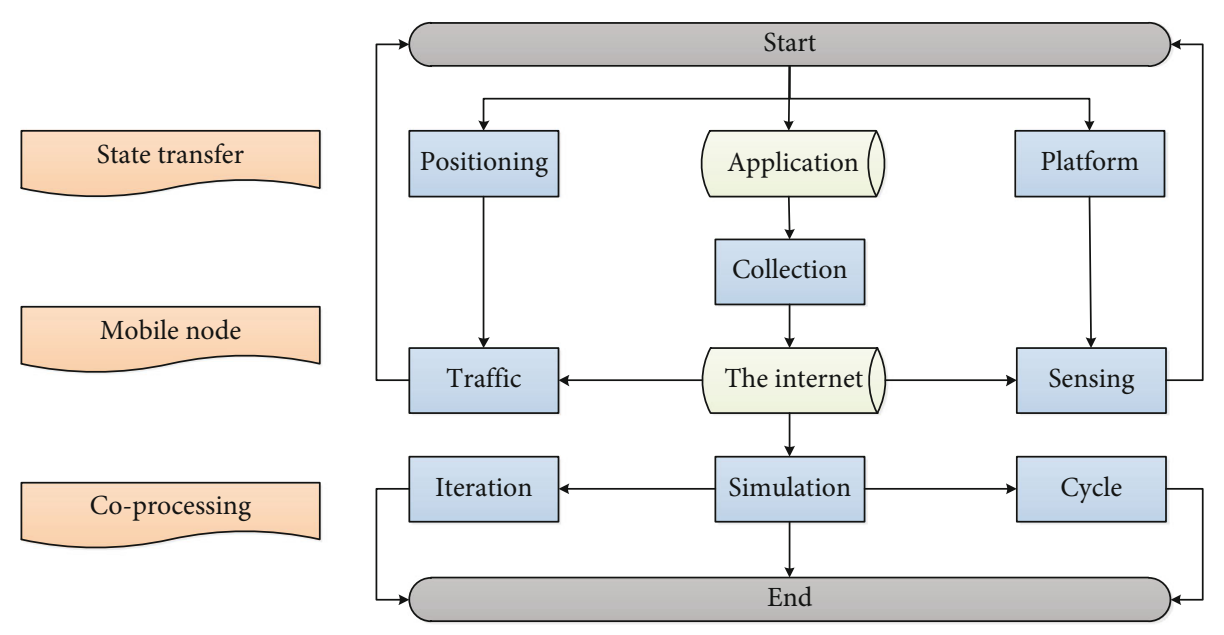

FIgURe 2: Traffic information collection algorithm flow.

3.3. Model Node Parameter Optimization. According to whether a beacon node is used in the positioning process, the positioning algorithm is divided into beacon-based positioning algorithm and beacon-free positioning algorithm. The former is in the positioning process. The beacon node is used as the reference point in the positioning, and the overall absolute coordinate system is generated after each node is positioned; the latter only cares about the relative position between the nodes, and no beacon node is needed in the positioning process. The nodes in the network are included in the coordinate system defined by themselves, and the adjacent coordinate systems are converted and merged in turn, and finally, the overall relative coordinate system is generated. First, a typical distance-vector exchange protocol is used to make all nodes in the network obtain the number of hops from the anchor node. In the second stage, after obtaining other anchor node positions and hop distances apart, the anchor node calculates the average network hop distance and then broadcasts it as a correction value to the network. The correction value adopts controllable flooding. The method propagates in the network, which means that a node only accepts the first correction value obtained, and discards all latecomers. This strategy ensures that most nodes can receive the correction value from the nearest anchor node. In large networks, the communication volume can be reduced by setting domain for the data packet. After receiving the correction value, the node calculates the distance to the anchor node according to the number of hops. Figure 3 shows the dependence of the network communication radius with the sample node. When the unknown node obtains the distance from 3 or more anchor nodes, the trilateration positioning is performed in the third stage.

The positioning equations obtained according to nonlinear characteristics usually need to be converted into linear equations first and then solved. The Taylor sequence expansion method is an effective method for solving nonlinear equations, which has the characteristics of high accuracy and strong robustness. Geometrically, each equation appears as a hyperbola. Among them, the object and environment models are used to describe the geographic environment of the area where the sensor node will be deployed or the spe-

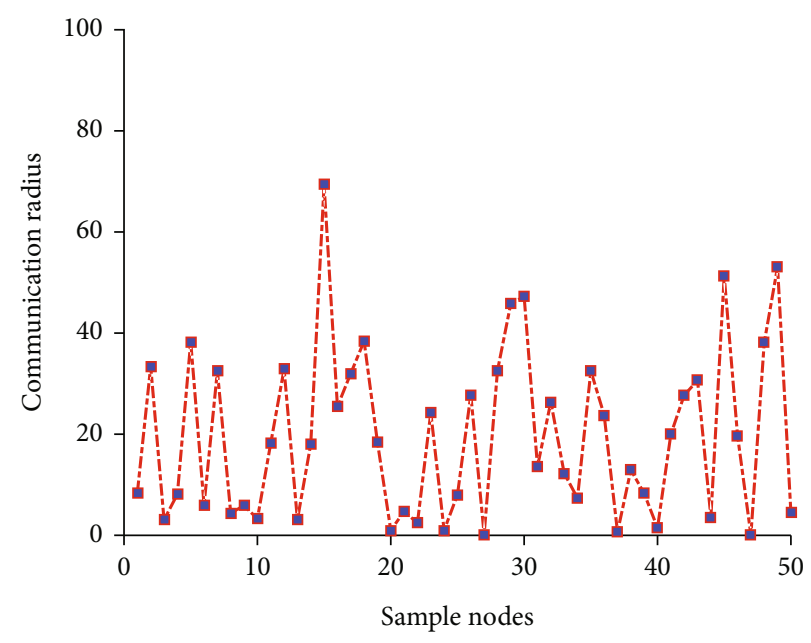

Figure 3: Dependency of network communication radius with sample nodes.

cial requirements of a certain area for node density. The deployed related parameters are used to define the sensing model, sensing range, communication range, and other coverage models of a single node, as well as other parameters. Figure 4 shows the framework of the traffic information collection model using wireless sensor network positioning technology. However, multipath effects and nonline-ofsight usually exist in the actual propagation environment of wireless sensor networks propagation, and the processing delay of the nodes will inevitably cause the error term in the measured.

On the other hand, because the number of anchor nodes involved in positioning generally exceeds the number of unknowns in the equations, the least squares method that can make full use of redundant information is an effective algorithm for solving such equations. Solving a system of nonlinear equations is equivalent to an unconstrained nonlinear optimization problem. First, it must be linearized. The Taylor series expansion method has been widely used in cellular positioning systems due to its high accuracy and 


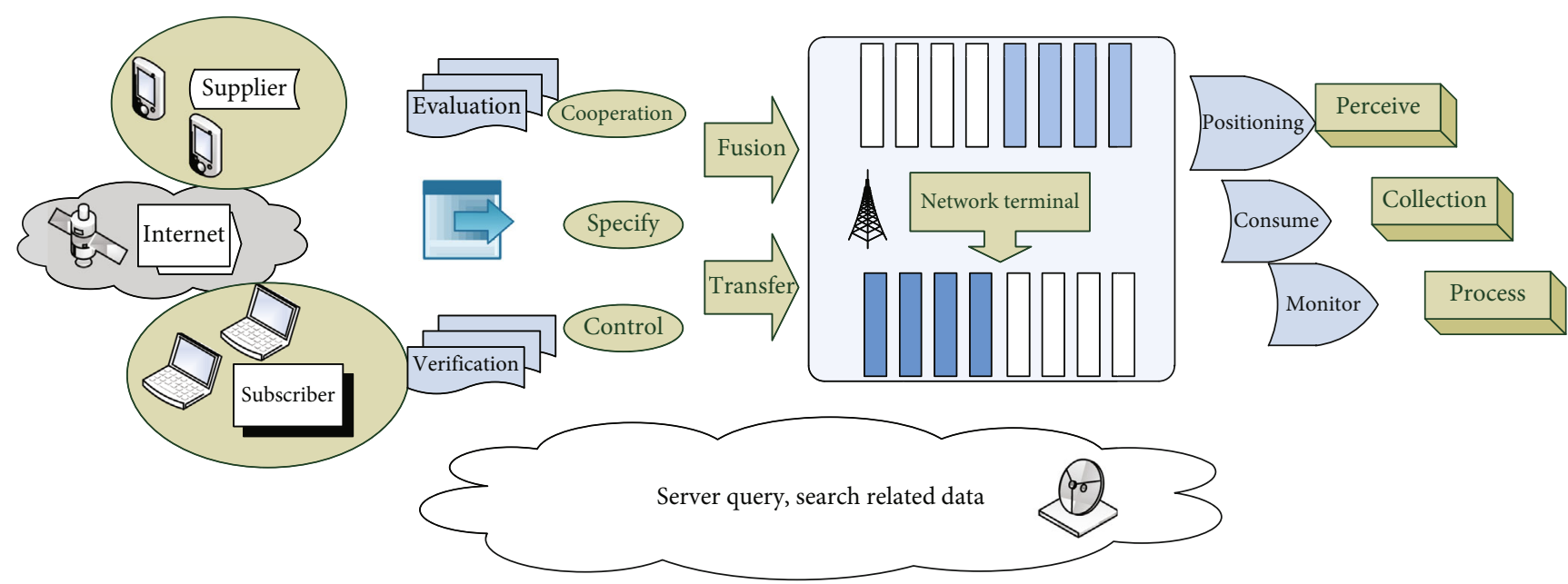

FIGURE 4: The framework of traffic information collection model using wireless sensor network positioning technology.

robustness. However, the Taylor sequence expansion method can obtain accurate calculation results, and an initial estimated position close to the actual position of the node to be measured is required to ensure algorithm convergence. The nonconvergence situation cannot be judged in advance. The initial estimated position close to the position is used to ensure the convergence of the algorithm, and the nonconvergence cannot be judged in advance. Therefore, after comparing the positioning performance of the algorithm and the Taylor sequence expansion method, this paper proposes an improved Taylor sequence expansion method that can be applied to outdoor WSN positioning systems and uses the extended Kalman filter method to optimize the algorithm performance.

\section{Analysis of Traffic Information Collection Model Based on WSN Positioning Technology}

4.1. Traffic Information Data Collection. Using Matlab, three scenarios were simulated when the throwing radius was $50 \mathrm{~m}$, and the grid inner ring radius $R=12.5 \mathrm{~m}$. The three scenarios are scenario 1, 250 nodes are randomly distributed; scenario 2, 500 nodes are randomly distributed; and scenario 3, 500 nodes, and the number of nodes in each grid is not less than 6 when distributed. Gateway nodes may be needed in the routing tree, which are distributed in a grid with fewer nodes. As the density of nodes increases, the distribution of nodes and cluster heads becomes more even, and there is basically no need for gateway nodes in the routing tree.

In order to study the total number of nodes in the wireless sensor network area is different, whether the optimal node communication radius changes, and the lowest node positioning error changes. Figure 5 shows the threedimensional fitting curve of the wireless sensor data packet. It shows the changes in node positioning error when the total number of nodes is 100,300 , and 500 , and the node communication radius changes from 5 to 50 . Using the dis-

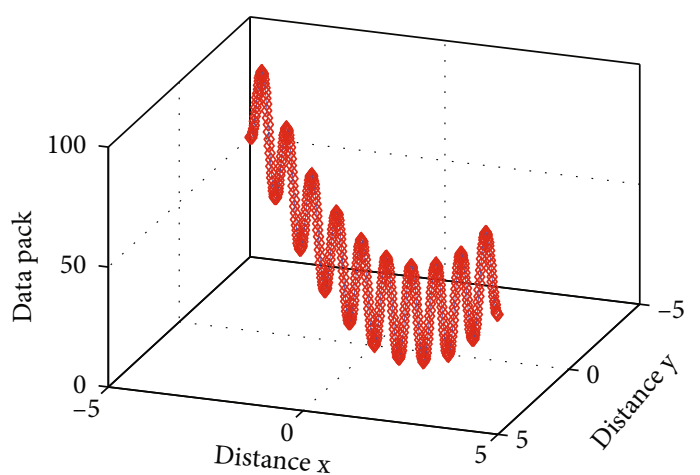

Figure 5: Three-dimensional fitting curve of wireless sensor data packet.

tance vector exchange protocol, the anchor node broadcasts its own position beacon containing the hop and accumulated hop distance (both initialized to 0 ) to the network. The node receiving the beacon only records the information of the anchor node with the smallest number of hops, accumulates the corresponding hop distance, and then increases the number of hops by 1 to forward the beacon. Finally, all nodes in the network can obtain the minimum cumulative hop distance of the corresponding anchor node. During the operation of the network, the nodes die one after another, resulting in a decrease in the number of nodes that can transmit data to the base station, that is, the number of effective nodes. In random topology, the coordinates of nodes are randomly generated, causing some nodes to be selected frequently and die quickly; in uniform topology, due to the uniform distribution of nodes, the probability of each node being selected when selecting the next hop has little difference, making the load more balanced which is distributed on each node. It shows that the networking and routing protocol proposed in this paper can balance the energy consumption of nodes and effectively extend the lifetime of the network. With the increase of the total number of nodes, the overall positioning error of the nodes is decreasing, and the optimal node communication radius is also 


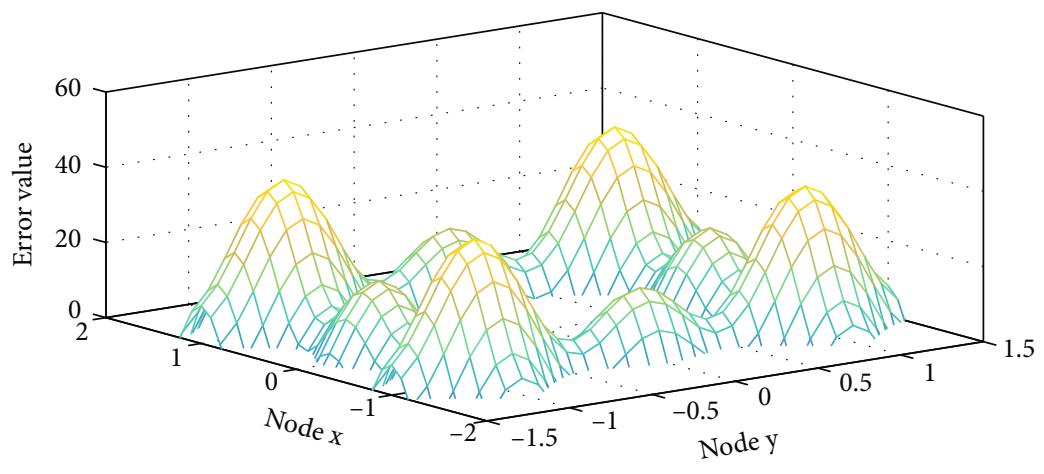

FIGURE 6: Three-dimensional error statistical distribution of traffic information.

changing. It shows the corresponding optimal node communication radius and corresponding lowest node positioning error value. Under the condition of different total number of nodes, there is an optimal node communication radius to reduce the positioning error to the lowest value. The positioning accuracy of the hop algorithm is the best. According to it, the optimal node communication radius can be selected under different total node numbers.

4.2. Wireless Sensor Network Positioning Simulation. The processing delay of the receiver refers to the time from the signal received by the antenna to the time it takes for the signal to be accurately decoded by the receiver. This delay is determined by the circuit of the receiver. It is usually regarded as a constant or fluctuates in a small range, and the error introduced by it can be ignored. Simulation conditions are three anchor nodes participating in TDOA measurement that set their position coordinates as $(0,0)$. Only the influence of TDQA measurement error on the positioning result is considered. The length of various information packets used in the simulation is 128 bits of control information packets when the base station scans and locates. The source data packet is 128 bits, the forwarding data packet is 512 bits, and the other control information packets are all 64 bits. The measurement error obeys the ideal Gaussian distribution, the mean value is 0 , and the standard deviation is $1 \mathrm{~m}, 2 \mathrm{~m}, 3 \mathrm{~m}, 4 \mathrm{~m}$, and $5 \mathrm{~m}$, respectively. The simulation results are shown in the following figure.

In order to make the simulation results more realistic and effective, the values are the average values obtained from 100 simulation results. We can get in the area of $1 \mathrm{~m} \times 1 \mathrm{~m}$, under the condition that the total number of nodes is 100 , when the number of anchor nodes is $8=12$. When the communication radius of the node is $R=22 \mathrm{~m}$. The positioning error of the node is small. Figure 6 shows the threedimensional error statistical distribution of traffic information. In the wireless sensor network area of $1 \mathrm{~m} \times 1 \mathrm{~m}$, the number of anchor nodes is 12 . First, we examine the performance of the improved DV-distance positioning algorithm under irregular and sparse network topologies. An anchor node is a node defined in an organization that has been added to the pipeline. This node is mainly used for node discovery. Every organization that joins the pipeline has at least one anchor node, and the node of an organization can find all the nodes of other organizations in the pipeline by searching for the anchor node. It can be seen that when the anchor node ratio is $10 \%$ and $20 \%$, the average positioning error of the improved algorithm will be significantly lower, which is about $10 \%$ to $30 \%$ lower than the original algorithm, indicating that the improved algorithm has better adaptability and stable positioning accuracy for low-density networks. Then, the ranging error varies from 5\% to $40 \%$ (relative to the communication radius), and the average positioning error before and after the algorithm is improved is compared.

The positioning error of the improved algorithm has been significantly reduced, and the degree of reduction is nearly $50 \%$. Figure 7 shows the accuracy fitting curve of the algorithm for different numbers of nodes. It can be seen that the improved DV-distance algorithm has better robustness and higher positioning accuracy. Compared with the average energy consumption of other algorithms, the node sleep waiting time is shorter. It can be seen from this that when time increases, the rate of increase in WSN energy consumption is much slower than that of other networks. When the energy consumption of the traffic information cluster collection algorithm increases, as the number of nodes increases, their energy consumption difference also increases rapidly, that is, it has better scalability. Under different node communication radii, the estimated distance between the two nodes is very different, which affects the positioning accuracy of the node. Therefore, the different communication radii of the nodes will lead to different positioning accuracy.

4.3. Example Application and Analysis. This section specifically gives the simulation results of the node positioning error when the number of anchor nodes, the communication radius of the node, and the total number of nodes change in the wireless sensor network area of $100 \mathrm{~m} \times 100 \mathrm{~m}$ and analyzes it. The number of anchor nodes in wireless sensor networks is different. Some parameters are defined as follows: the number of unknown nodes $n=30$; the number of reference nodes $m=4$; the node communication radius $R=5 \mathrm{~m}$; the number of iterations $k$; the empirical threshold based on accuracy requirements $s=0.0001$. The unknown nodes are randomly arranged in a rectangular area of $9 \mathrm{~m} \times 9 \mathrm{~m}$. For the convenience of observation, the reference nodes are, respectively, arranged at the four vertices of the quadrilateral area. Here, the positioning error rate is used to 


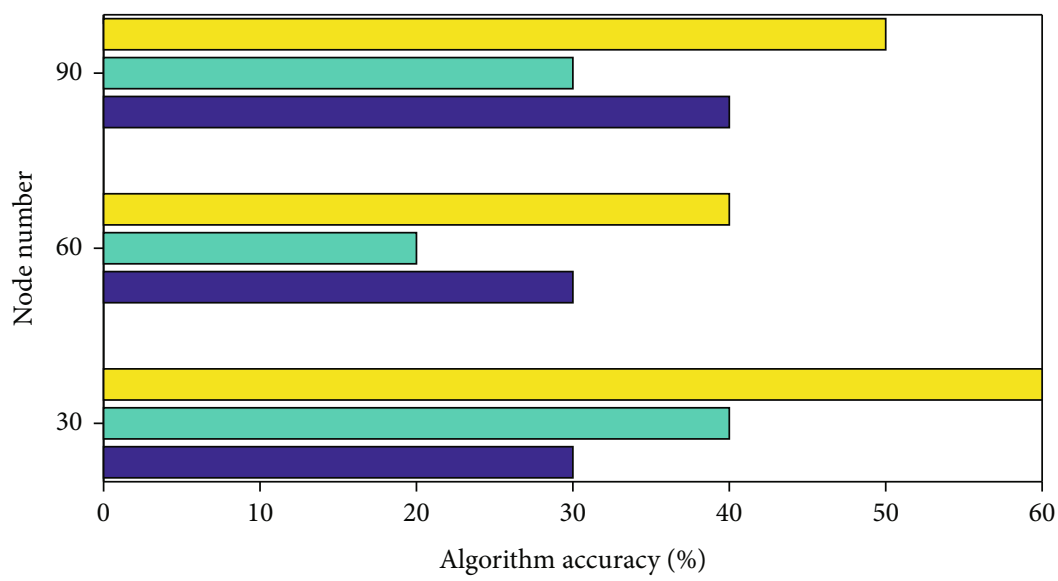

Reference [20]

$\square$ Reference [21]

$\square$ This work

FIGURE 7: Algorithm accuracy fitting curve of different number of nodes.

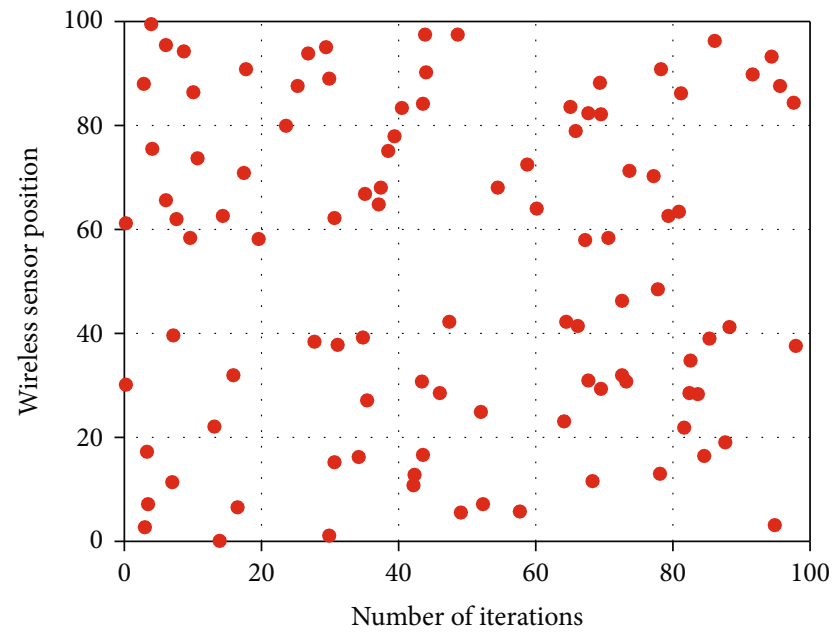

Figure 8: Two-dimensional scatter plot of wireless sensor positioning with the number of iterations.

measure the performance of the positioning algorithm. A smaller positioning error indicates a higher accuracy of the positioning algorithm. In order to facilitate comparison, we first set $k=60$ to compare the positioning situation before and after improvement.

In a certain area of the sensing area, the total number of different sensor nodes also affects DV. As shown in the figure, under the same node communication radius, the total number of nodes is different. Figure 8 shows a twodimensional scatter plot of wireless sensor positioning with the number of iterations. The number of nodes in an average hop will vary greatly. If the number of nodes is too small, there may be no nodes within the communication range of the anchor node. The basic idea of serial interference cancellation technology is to adopt a step-by-step interference elimination strategy, in which the user is judged one by one in the received signal, and after the amplitude is

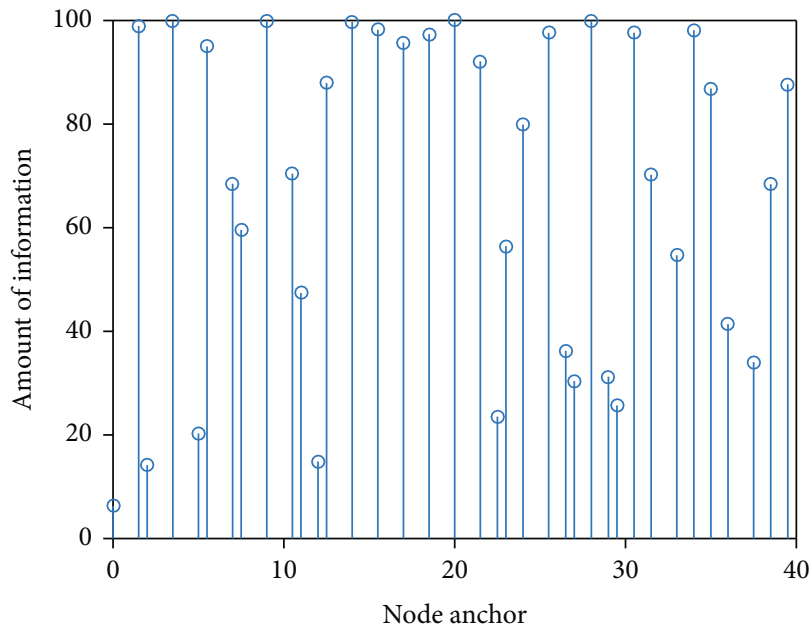

FIGURE 9: Matchstick graph of traffic information varying with anchor nodes.

restored, the multiple access interference generated by the user signal is subtracted from the received signal and the residual. The next user makes a decision again and repeats the operation until all the multiple access interference is eliminated. Other nodes cannot receive the anchor node's information, resulting in the situation where the node cannot be located. Increasing $k$ and $s$, we calculate the positioning error rate of each iteration before and after the improvement and get the relationship between the number of iterations and the positioning error. As shown in the figure, to achieve a positioning error of $0.10 \mathrm{~m}$, the improved iterative algorithm requires more than 220 iterations to get it, while the improved iterative algorithm only needs less than 10 iterations to achieve the same accuracy. The computational workload of the algorithm is reduced by more than 20 times.

Figure 9 shows the matchstick graph of the amount of traffic information varying with anchor nodes. In figure, 


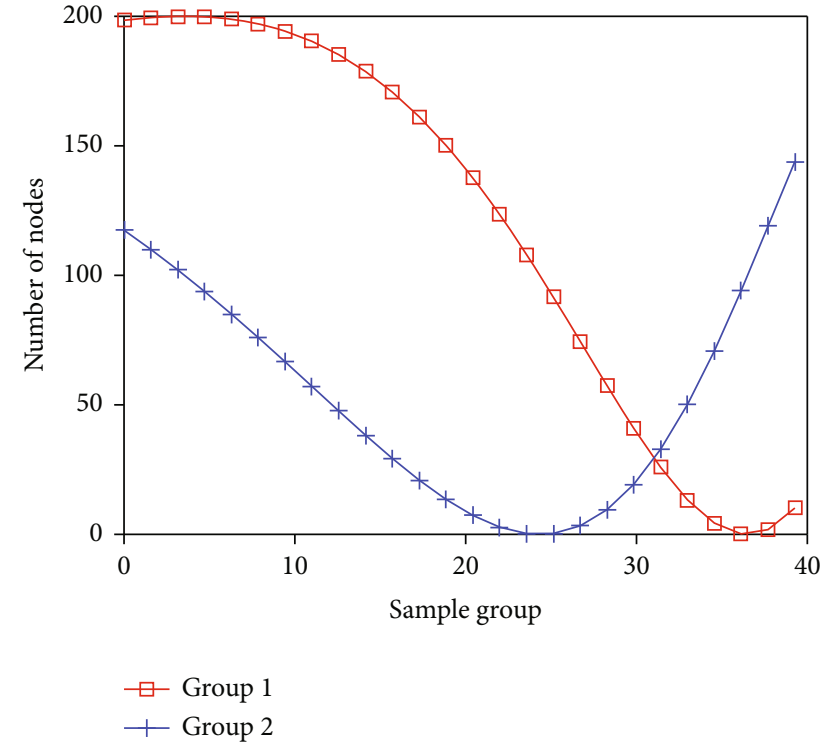

FIGURE 10: Comparison of distribution curves of communication nodes in different sample groups.

given the number of anchor nodes, the node positioning error is shown when the node communication radius $R$ changes from 10 to 50 . It can be seen that as the number of anchor nodes gradually increases (when the number of anchor nodes is changed from 6 to 12), the positioning error of the node is correspondingly reduced; when the number of anchor nodes is different, when the communication radius of the node changes from 10 to 20 . The node positioning error is rapidly reduced and reaches the minimum value. As the communication radius of the nodes continues to increase, the positioning error of the nodes also begins to increase, indicating that when the anchor nodes are different and the total number of nodes is 100 , there is an optimal ruler value. The positioning accuracy of the node can be seen: when the communication radius of the node and the total number of nodes are different, the number of anchor nodes is about 12 . The positioning error of the node can be reduced to a smaller value; when the number of anchor nodes changes from 3 to 10 . The positioning error of the node decreases rapidly: as the number of anchor nodes continues to increase, the positioning error of the node decreases, but the amplitude tends to be flat.

From the above analysis, it can be seen that when the number of anchor nodes reaches a certain number, its influence on the node positioning error is reduced. Figure 10 shows the comparison of the distribution curves of communication nodes in different sample groups. It shows that when the node communication radius is different from the total number of nodes, the node positioning error is basically close to the minimum when the number of anchor nodes is 12. This paper presents the change of node positioning error when the number of anchor nodes is different under the condition that the total number of nodes is determined. The node communication radius $R$ changes from 10-50, and the node's positioning error changes.

\section{Conclusion}

When using the traditional positioning algorithm for positioning in this paper, it will encounter the problem of overdetermined nonlinearity of the positioning equation, and iterative method is needed to solve it. Based on this, this paper proposes a hybrid positioning algorithm, successfully simplifies the problem of solving iterative positioning equations, successfully reduces the algorithm complexity, and reduces system power consumption. At the same time, in response to the problem of high system power consumption, this article also proposes to use Zigbee protocol instead of traditional WiFi protocol to develop a positioning system, thereby effectively reducing system power consumption, increasing system life, and improving the scope of system application. 6G has extremely high requirements for energy efficiency ratio, which requires encoding, modulation, sending, and receiving processing in an energy-saving manner to achieve a balance between the transmitted energy and the required processing energy. Backscatter communication using radio frequency power for connection and calculation may be an effective way to achieve ultralow power communication. Backscatter communication can obtain energy from the environment and radio frequency waveforms, which can extend the life of IoT nodes. Regarding the node location problem in wireless sensor networks as the dual problem of target tracking, a node location solution based on virtual beacon points is proposed, which is different from the existing location algorithm based on fixed beacon points. It uses the nonlinear least squares optimization method to solve the minimum cost function composed of energy comparison to realize node self-positioning. This algorithm improves the positioning accuracy without increasing the communication volume and computational complexity of the original algorithm. The simulation results show that under the same conditions, the positioning accuracy of the algorithm proposed in this paper is improved by $5-10 \%$.

\section{Data Availability}

The data used to support the findings of this study are available from the corresponding author upon request.

\section{Conflicts of Interest}

The authors declare that they have no known competing financial interests or personal relationships that could have appeared to influence the work reported in this paper.

\section{References}

[1] M. A. Matin and M. M. Islam, "Overview of wireless sensor network," Wireless Sensor Networks-Technology and Protocols, vol. 2, pp. 1-3, 2019.

[2] N. Jing, "Application of wireless sensor network in urban intelligent traffic information acquisition," Automatic Control and Computer Sciences, vol. 52, no. 5, pp. 431-438, 2018.

[3] M. Mbodila, E. Obeten, and I. Bassey, "Implementation of novel vehicles' traffic monitoring using wireless sensor 
network in South Africa," Communication Software and Networks, vol. 5, pp. 282-286, 2019.

[4] J. Jiang, H. Wang, X. Mu, and S. Guan, "Logistics industry monitoring system based on wireless sensor network platform," Computer Communications, vol. 155, pp. 58-65, 2020.

[5] A. U. Rahman, A. Alharby, H. Hasbullah, and K. Almuzaini, "Corona based deployment strategies in wireless sensor network: a survey," Journal of Network and Computer Applications, vol. 64, pp. 176-193, 2016.

[6] J. Yick, B. Mukherjee, and D. Ghosal, "Wireless sensor network survey," Computer Networks, vol. 52, no. 12, pp. 22922330, 2018.

[7] J. Zhou, C. L. P. Chen, L. Chen, and W. Zhao, "A usercustomizable urban traffic information collection method based on wireless sensor networks," IEEE Transactions on Intelligent Transportation Systems, vol. 14, no. 3, pp. 11191128, 2013.

[8] M. Sugano, T. Kawazoe, Y. Ohta, and M. Murata, "Indoor localization system using RSSI measurement of wireless sensor network based on ZigBee standard," Wireless and Optical Communications, vol. 538, pp. 1-6, 2018.

[9] Y. Liu, X. Mao, Y. He, K. Liu, W. Gong, and J. Wang, "CitySee: not only a wireless sensor network," IEEE Network, vol. 27, no. 5, pp. 42-47, 2013.

[10] S. Vançin and E. Erdem, "Design and simulation of wireless sensor network topologies using the ZigBee standard," International Journal of Computer Networks and Applications, vol. 2, no. 3, pp. 135-143, 2019.

[11] Y. Jo, J. Choi, and I. Jung, "Traffic information acquisition system with ultrasonic sensors in wireless sensor networks," International Journal of Distributed Sensor Networks, vol. 10, no. 5, Article ID 961073, 2014.

[12] M. M. Nabeel, M. F. el Deen, and S. El-Kader, "Intelligent vehicle recognition based on wireless sensor network," International Journal of Computer Science, vol. 10, no. 4, pp. 164174, 2019.

[13] I. Oppermann, L. Stoica, A. Rabbachin, Z. Shelby, and J. Haapola, "UWB wireless sensor networks: UWEN - a practical example," IEEE Communications Magazine, vol. 42, no. 12, pp. S27-S32, 2004.

[14] C. F. Zhen, W. Liu, Y. Liu, and A. Yan, "Energy-efficient sleep/wake scheduling for acoustic localization wireless sensor network node," International Journal of Distributed Sensor Networks, vol. 10, no. 2, Article ID 970524, 2014.

[15] S. S. Jan, L. T. Hsu, and W. M. Tsai, "Development of an indoor location based service test bed and geographic information system with a wireless sensor network," Sensors, vol. 10, no. 4, pp. 2957-2974, 2010.

[16] M. Cardei and D. Z. Du, "Improving wireless sensor network lifetime through power aware organization," Wireless Networks, vol. 11, no. 3, pp. 333-340, 2005.

[17] A. M. Badescu and L. Cotofana, "A wireless sensor network to monitor and protect tigers in the wild," Ecological Indicators, vol. 57, pp. 447-451, 2015.

[18] M. C. Chen, S. Q. Lu, and Q. L. Liu, "Uniqueness of weak solutions to a Keller-Segel-Navier-Stokes system," Applied Mathematics Letters, vol. 121, article 107417, 2021.

[19] M. C. Chen, S. Q. Lu, and Q. L. Liu, "Uniqueness of weak solutions to a Keller-Segel-Navier-Stokes model with a logistic source," Applications of Mathematics, pp. 1-9, 2021.
[20] P. Wang and G. Tu, "Localization algorithm of wireless sensor network based on matrix reconstruction," Computer Communications, vol. 154, pp. 216-222, 2020.

[21] W. Chen, L. Gao, Z. Chai, Z. Chen, and S. Tu, “An intelligent guiding and controlling system for transportation network based on wireless sensor network technology," Computer and Information Technology, vol. 5, pp. 810-814, 2019.

[22] Z. Wu, H. Chu, Y. Pan, and X. Yang, "Bus priority control system based on wireless sensor network (WSN) and zigbee," in 2006 IEEE International Conference on Vehicular Electronics and Safety, Shanghai, China, December 2006.

[23] Z. Zheng, J. Lv, C. Li et al., "The improved indoor localisation algorithm based on wireless sensor network," International Journal of Computational Science and Engineering, vol. 15, no. 3-4, pp. 322-329, 2017.

[24] C. Gong, Y. Hu, J. Gao, Y. Wang, and L. Yan, “An improved delay-suppressed sliding-mode observer for sensorless vector-controlled PMSM," IEEE Transactions on Industrial Electronics, vol. 67, no. 7, pp. 5913-5923, 2020.

[25] Y. Liu, B. Zhang, Y. Feng et al., "Development of $340-\mathrm{GHz}$ transceiver front end based on GaAs monolithic integration technology for $\mathrm{THz}$ active imaging array," Applied Sciences, vol. 10, no. 21, p. 7924, 2020.

[26] J. Yang, S. Xiao, B. Jiang, H. Song, S. Khan, and S. . Islam, "Cache-enabled unmanned aerial vehicles for cooperative cognitive radio networks," IEEE Wireless Communications, vol. 27, no. 2, pp. 155-161, 2020.

[27] Z. Cai, Z. He, X. Guan, and Y. Li, "Collective data-sanitization for preventing sensitive information inference attacks in social networks," IEEE Transactions on Dependable and Secure Computing, p. 1, 2016.

[28] J. Chen, C. Du, Y. Zhang et al., "A clustering-based coverage path planning method for autonomous heterogeneous UAVs," IEEE Transactions on Intelligent Transportation Systems, 2021.

[29] W. Wang, N. Kumar, J. Chen et al., "Realizing the potential of the internet of things for smart tourism with $5 \mathrm{G}$ and AI," IEEE Network, vol. 34, no. 6, pp. 295-301, 2020.

[30] C. Peng and Z. Yu, "Modeling analysis for positioning error of mobile Lidar based on multi-body system kinematics," Intelligent automation and soft computing, vol. 25, no. 4, pp. 827-834, 2019.

[31] T. Zhou, B. Lian, S. Yang, Y. Zhang, and Y. Liu, "Improved GNSS cooperation positioning algorithm for indoor localization," Computers, Materials and Continua, vol. 56, no. 2, pp. 225-245, 2018.

[32] J. Wang, X. Qiu, and Y. Tu, “An improved MDS-MAP localization algorithm based on weighted clustering and heuristic merging for anisotropic wireless networks with energy holes," Computers, Materials and Continua, vol. 58, no. 2, pp. 227244, 2019.

[33] M. Kanthimathi, R. Amutha, and K. S. Kumar, "Energy efficiency analysis of differential cooperative algorithm in wireless sensor network," Cluster Computing, vol. 22, no. S4, pp. 98379845, 2019.

[34] J. Wen, J. Yang, B. Jiang, H. Song, and H. Wang, "Big data driven marine environment information forecasting: a time series prediction network," IEEE Transactions on Fuzzy Systems, vol. 29, no. 1, pp. 4-18, 2021.

[35] S. C. Chu, Z. G. Du, and J. S. Pan, "Symbiotic organism search algorithm with multi-group quantum-behavior communication scheme applied in wireless sensor networks," Applied Sciences, vol. 10, no. 3, p. 930, 2020. 